\title{
Pictorial Narratives and Temporal Refinement
}

\author{
Tim Fernando \\ Trinity College Dublin
}

\begin{abstract}
Refinements are proposed to the default reading of two successive pictures $p, p^{\prime}$ as $p$ and then $p^{\prime}$. The refinements are based on the Aristotelian dictum, no time without change, and the principle of inertia, no change without force, guided by the adage, a picture's worth a thousand words. Words describing pictures are formalized as predicates, some stative and some non-stative (expressing forces), and interpreted (in either case) over strings qua models, subject to finite-state projections supporting variable granularity. A form of string iconicity is explored, with an eye to more transparent representations.
\end{abstract}

Keywords: pictorial narrative, temporal refinement, stative, force, string iconicity

\section{Introduction}

At the root of viewpoint-centered propositions in recent work on the semantics of pictorial narratives (Abusch 2013, 2014; Abusch \& Rooth 2017; Rooth \& Abusch 2018; Maier \& Bimpikou To appear: among others) is an understanding of pictures in terms of projections (e.g., Hagen 1986). Stepping beyond any single picture within a narrative, we may ask

(Q) Is there a projective account of temporal progression in pictorial narratives?

A positive answer to (Q) is presented below, in which a sequence $p_{1} p_{2} \cdots p_{n}$ of pictures $p_{i}$ constituting a pictorial narrative is turned into a string of boxes not unlike the Discourse Representation Structures of Kamp \& Reyle (1993). The simple case of representing $p_{1} p_{2}$ as (D) follows Dowty (1986)'s rule of temporal succession, adopted by Abusch (2014) for a default reading specifying two stretches of time, one with $p_{1}$, followed by one with $p_{2}$.

(D)

$$
\begin{array}{|l|l|l|l|l|l}
\hline p_{1} & p_{2}
\end{array} \quad \text { (default progression: ' } p_{1} \text { and then } p_{2} \text { ') }
$$

Under an alternative reading $(\mathbf{N}), p_{1}$ and $p_{2}$ describe one and the same stretch of time - or one box.

$(\mathbf{N})$

$$
p_{1}, p_{2} \quad \text { (no progression: ' } p_{1} \text { and simultaneously } p_{2} \text { ') }
$$


The idea is that temporal boundaries are marked by boxes, each assumed to carve out an interval of time, abutting intervals from adjacent boxes, arranged so that the box to the left is earlier than that to the right. Now, if we assume that $p_{1}$ and $p_{2}$ are stative, as Abusch (2014) does, then the inertiality of statives (Dowty 1986) suggests that in the default reading $(\mathbf{D}), p_{1}$ persists forward to the next box, and $p_{2}$ persists backward to the previous box, unless a force intervenes. That is, in the absence of an intervening force, inertia transforms (D) into (I).

$$
\left.\begin{array}{l|l|l}
\hline p_{1}, p_{2} & p_{1}, p_{2} \\
\hline
\end{array} \quad \text { (inertia: stutter } \alpha \alpha\right)
$$

(I) consists of two temporal stretches, both featuring $p_{1}$ and $p_{2}$. As statives are cumulative, we can merge these two stretches into one (effectively deleting a time without change). Progression by default, (D), has turned into no progression, (N). This is welcome only in cases where the default should be overriden (not where the default applies). The moral, it would appear, is that if (D) and (N) are distinct, it is because a barrier between $p_{1}$ and $p_{2}$ is erected by some force. But what force? Can we make this reasoning rigorous, and develop it further? Or should we put $(\mathbf{D}),(\mathbf{N})$ and (I) away, and not bother?

\subsection{Iconicity, sequence, transformation and representation}

The default reading (D) of $p_{1} p_{2}$, traced above to Dowty (1986), is widely associated with iconicity, understood as resemblance between the form of a sign and its meaning. "Iconicity of sequence" describes cases where "the sequence of forms conforms to the sequence of experience" (Perniss \& Vigliocco 2014: 10). For pictorial representation, the significance of resemblance is challenged by Greenberg (2013), crediting instead the view of depiction as geometrical transformation (e.g., Hagen 1986). Transformations of representations, pictorial and otherwise, lead to "the mystery of deduction" (Shin 2015) for sufficiently complex transformations of representations of the premise to representations of the conclusion. That complexity is minimised in "an absolutely transparent representation" where "we could read off all of the logically implied conclusions from the given information"

With a transparent representation, we may see that the conclusionrepresentation is a (physical or literal) part of the premise-representation without any manipulation, or, if transformations are needed, then finding a pathway from one representation to another representation is trivial or mechanical. (Shin 2015: 51-52)

A conclusion that is "part of" a premise can be drawn from eliminating an occurrence of the binary connective $\wedge$ representing and. 
Pictorial narratives and temporal refinement

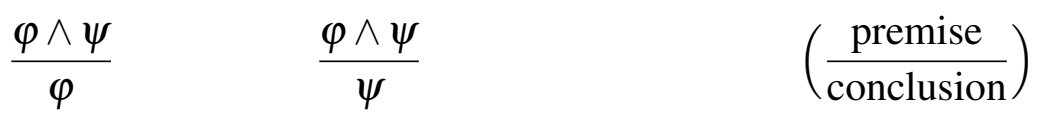

Conjunction elimination lies behind the representation of events in Davidson (1967), about which, more later. For now, consider the question

(Q2) Can pictures and logical formulas combine in a system of transparent representations?

Automating translations between pictures and language is a challenging task; it would be no mean feat to demonstrate (Q2) can be answered affirmatively for a nontrivial range of pictures and logical formulas (and a useful notion of transparency).

The present paper provides no such demonstration, leaving the effortlessness with which humans routinely mix pictures and language a mystery. Short of explaining that mystery away, we focus more modestly on how logical formulas might serve as stepping stones for understanding time in pictorial narratives. In particular, logical formulas are applied to make sense of the strings (D), (N) and (I) of boxes above.

\subsection{Strings and the big picture}

The formulas are constructed from a finite set $A$, each element $a \in A$ of which labels a distinct unary predicate symbol $P_{a}$. It is natural to construe a string $s=\alpha_{1} \cdots \alpha_{n}$ of subsets $\alpha_{i}$ of $A$ as interpreting each $P_{a}$ as a subset $\left[\left[P_{a}\right]\right]$ of the set

$$
[n]:=\{1,2, \ldots, n\}
$$

of integers from 1 to $n$, namely, the set

$$
\left[\left[P_{a}\right]\right]:=\left\{i \in[n] \mid a \in \alpha_{i}\right\}
$$

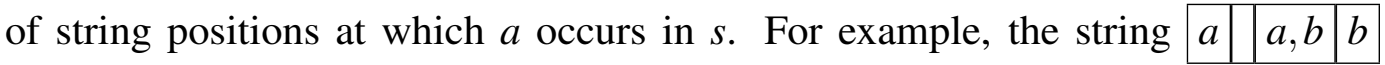
interprets $P_{a}$ as $\left[\left[P_{a}\right]\right]=\{1,3\}$ and $P_{b}$ as $\left[\left[P_{b}\right]\right]=\{3,4\}$. Let $2^{A}$ be the power set of $A$ (i.e., the set of subsets of $A$ ), and let us call a string over the alphabet $2^{A}$ an $A$-string. An $A$-string $\alpha_{1} \cdots \alpha_{n}$ can be reconstructed from $n$ and the subsets $\left[\left[P_{a}\right]\right]$ of $[n]$ indexed by $A$ through the equations

$$
\alpha_{i}=\left\{a \in A \mid i \in\left[\left[P_{a}\right]\right\} \quad \text { for all } i \in[n] .\right.
$$

To describe strings in predicate logic, it is customary to introduce a binary relation symbol $S$ (for successor), and repackage an $A$-string $\alpha_{1} \cdots \alpha_{n}$ as an $A$-model $\left\langle[n], S_{n},\left[\left[P_{a}\right]\right]_{a \in A}\right\rangle$ with universe/domain/carrier set $[n]$, interpreting $P_{a}$ as the subset $\left[\left[P_{a}\right]\right]$ of $[n]$ (for each $a \in A$ ) and $S$ as the +1 relation

$$
S_{n}:=\{(1,2), \ldots,(n-1, n)\}=\{(i, i+1) \mid i \in[n-1]\}
$$


on $[n]$. For instance, $S_{4}=\{(1,2),(2,3),(3,4)\}$.

It is tempting to describe an $A$-string $\alpha_{1} \cdots \alpha_{n}$ as $n$ successive snapshots, starting with $\alpha_{1}$, followed by $\alpha_{2}, \ldots$ ending with $\alpha_{n}$. But let us resist confusing a box $\alpha_{i}$ with a picture $p_{i}$, not to mention the sequence $\alpha_{1} \cdots \alpha_{n}$ with a pictorial narrative $p_{1} \cdots p_{n}$. In (D), (N) and (I) above, a picture $p$ is treated as an element of $A$ that may go into a box $\alpha$. Under the aforementioned projective conception of $p$ as the output of a function $\pi$ that is fed a scene $\sigma$ and a viewpoint $v$

$$
p=\pi(\sigma, v),
$$

a box $\alpha$ represents some $\sigma$ for which

$$
\text { ' } p \in \alpha \text { ' suggests } \quad p=\pi(\sigma, v) \text { for some viewpoint } v \text {. }
$$

To make this suggestion precise for a fixed function $\pi$, let us represent $\sigma$ as the set

$$
\sigma^{\pi}:=\{(p, v) \mid \pi(\sigma, v)=p\} \cup\{p \mid \pi(\sigma, v)=p \text { for some } v\}
$$

which, given any finite sets $P$ and $V$ of pictures and viewpoints respectively, we can turn into a finite set by intersection

$$
\sigma^{\pi}[P, V]:=\sigma^{\pi} \cap(P \cup(P \times V)) .
$$

Applying the transformation $\lambda \sigma \cdot \sigma^{\pi}[P, V]$ componentwise to a string $\sigma_{1} \cdots \sigma_{n}$ of scenes yields the $(P \cup(P \times V))$-string

$$
\alpha_{1} \cdots \alpha_{n} \text { where } \alpha_{i}:=\sigma_{i}^{\pi}[P, V]
$$

which for every $p \in P$, satisfies the predicate logic sentence

$$
\left.\forall x\left(P_{p}(x) \equiv \bigvee_{v \in V} P_{(p, v)}(x)\right) \quad \text { (biconditional } \equiv, \text { disjunction } \bigvee\right)
$$

saying $p$ occurs at and only at string positions where $(p, v)$ does for some $v \in V$. $^{1}$ The sets $\sigma^{\pi}[P, V]$ depend on not just $P$ and $V$ but also the projection $\pi$, incorporating

1 Just as the proposition expressed by the sentence

$$
\text { you are hungry }
$$

varies with the addressee and utterance time, the particular object depicted by a picture $p$ (of say, an empty cup) varies with $\pi, \sigma$ and $v$ as we may have

$$
\pi(\sigma, v)=p=\pi^{\prime}\left(\sigma^{\prime}, v^{\prime}\right) \text { even though } \pi \neq \pi^{\prime} \text { or } \sigma \neq \sigma^{\prime} \text { or } v \neq v^{\prime} .
$$

Pairing $p$ with $v$ sharpens the predicate $P_{p}$ to $P_{(p, v)}$, as asserted by (1). More on (1) in $\S \S 2.2-3$ below. 
parameter settings suppressed in the notation $\pi(\sigma, v)$. Deviating from any choice of $\pi$ allows for artistic license, taken, for instance, by a painting $p$ that blends various times. But literal meaning has always come ahead of figurative meaning in formal semantics, so should artistic license not wait? Positions differ ...

Be that as it may, we will not dwell on the choice of $\pi$ below, focusing rather on the temporal relations between pictures in a narrative. Kamp (2013) expresses a guiding intuition about natural language that arguably applies to a narrative with pictures in place of sentences

when we interpret a piece of discourse - or a single sentence in the context in which it is being used — we build something like a model of the episode or situation described; and an important part of that model are its event structure, and the time structure that can be derived from that event structure (Kamp 2013: 13).

That event structure consists, in practice, of finitely many events (roughly, those mentioned in the discourse), marking out a finite set of temporal stretches (far coarser than the real line $\mathbb{R}$, often used for time). This temporal structure is formulated below using strings such as $(\mathbf{D}),(\mathbf{N})$ and (I) above, on the understanding that boxes are arranged in chronological order. An assumption essential for the discreteness of time (justifying talk of the next or previous temporal stretch) is the requirement that the set $A$ of labels $a$ of predicates $P_{a}$ be finite (explaining the intersection of $\sigma^{\pi}$ above with the finite set $P \cup(P \times V)$ of labels). Having bounded the granularity by requiring $A$ be finite, we can coarsen the granularity by reducing $A$ to some subset of it, or refine that granularity by moving to a larger finite set $A^{\prime}$ of labels. This enlargement can be carried out in any number of ways, not limited to adding more pictures $p$ or viewpoints $v$ (outside $P$ or $V$, respectively) for labels $p$ and $(p, v)$. For instance, we might represent the pixelmap associated with a picture $p$ as a finite set of labels $c(z)$ classifying a region $z$ of space as $c$. Or a label $(p, z, x)$ might annotate a picture $p$ with a discourse referent $x$ for an entity depicted in some region $z$ of $p$ to facilitate co-reference across pictures (Abusch 2013). Or a label see $(z, p)$ might specify the picture $p$ an agent $z$ sees, as in free perception hidden operators (Abusch $\&$ Rooth 2017). Just as there is no limit to unary predicate symbols that one can dream up, so too is there no limit to labels, which we can collect in some large infinite set $\Theta$. The idea then is to form $A$-strings, where $A$ is a finite subset of $\Theta$. The set $\operatorname{Fin}(\Theta)$ of finite subsets of $\Theta$ serves as the basis of a projective system of variable temporal granularity (Fernando 2016, 2019), reviewed below.

With respect to the discussion of the strings (D), (N) and (I) above, we draw on insights from event semantics to develop the following claims in section 2 .

(a) The derivation of (N) from (D) above rests on the absence of forces at play 
- an assumption unwarranted in cases of change (for progression), but appropriate for portraying a static background.

(b) Forces are suggested, if not depicted outright, in a picture or a succession of pictures.

(c) Inertia is not all-or-nothing (nor is a picture, in general, wholly stative or wholly non-stative): some pieces of a picture may persist, others may not.

(d) Breaking a picture $p$ up into a finite set $\alpha$ of predicates facilitates a separation of stative pieces from non-stative ones expressing forces.

Eyeing transparent representations, we proceed in section 3 to flesh out

(e) Granularity can be varied via projections that recognize gaps between boxes and the difference between statives and non-statives (with non-stative pieces from $p_{1}$ or $p_{2}$, if they exist, blocking the passage from $(\mathbf{D})$ to $(\mathbf{N})$ ).

We make a nod at the end to other mechanisms that override the default reading $(\mathbf{D})$, deploying representations that go beyond $A$-strings (e.g., Asher \& Lascarides 2003; Cooper \& Ginzburg 2015).

\section{A picture as a thousand words: stative and non-stative pieces}

How are we to turn a pictorial narrative $p_{1} \cdots p_{n}$ of pictures into a sequence $\alpha_{1} \cdots \alpha_{k}$ of boxes representing what the narrative is about? We can start by breaking a picture $p$ into pieces, which we then put in a box $\alpha$ - any two pieces composing conjunctively to describe $p$ (and decomposing by conjunction elimination). Pixelmaps provide familiar examples, with which we may take liberties, replacing low-level information such as black $(201,93)$ with more abstract descriptions such as smilingPat. Bringing other pictures $p^{\prime}$ into the mix complicates matters with questions about time and change. On the question of the aspectual type of pictures and its ramifications for temporal progression, Abusch claims

Some examples suggest that pictures can have stative aspect and that such pictures do not advance time, paralleling the situation in natural language. However, we show on the basis of formal semantics that all pictures have stative informational content, so that a construction rule for pictorial discourse representations cannot be sensitive to aspect. This suggests that the temporal interpretation rule for pictorial narratives involves invariant temporal progression. (Abusch 2014: 9). 
Pictorial narratives and temporal refinement

This claim is re-examined in this section, with pictures broken into pieces collected in $A$, and pictorial narratives interpreted in terms of $A$-strings that represent time in accordance with the Aristotelian dictum

(Ntc) no time without change

and the principle of inertia

(Inr) no change without force.

Recall that (Ntc) and (Inr) figure in the discussion of (D), (N) and (I) in the Introduction; very roughly, (Inr) takes us from (D) to (I), while (Ntc) takes us from (I) to (N). Our aim now is to make this rough account rigorous.

\subsection{Statives and strings}

Statives are central to Dowty's well-known hypothesis that

the different aspectual properties of the various kinds of verbs can be explained by postulating a single homogeneous class of predicates - stative predicates - plus three or four sentential operators or connectives (Dowty 1979: 71)

Insofar as Dowty's aspectual calculus or related temporal representations are built from statives, it is not surprising that "all pictures have stative information content." But of course, Abusch (2014) has more in mind than the mere presence of statives in these representations. A predicate $\mathrm{P}$ on intervals is homogeneous if $\mathrm{P}$ holds of an interval $I$ precisely if for every element $t$ of $I$, P holds of the interval $\{t\}$

$$
\mathrm{P}(I) \Longleftrightarrow(\forall t \in I) \mathrm{P}(\{t\})
$$

Homogeneous predicates carry certain entailments, such (2) and (3).

(2) Pat slept from $11 \mathrm{pm}$ to $7 \mathrm{am} \Longrightarrow$ Pat slept from midnight to 3am

(3) Pat slept from $11 \mathrm{pm}$ to $3 \mathrm{am}$ and from $2 \mathrm{am}$ to $7 \mathrm{am} \Longrightarrow$ Pat slept from $11 \mathrm{pm}$ to $7 \mathrm{am}$

Let us call a predicate $\mathrm{P}$ on intervals segmented if for all intervals $I$ and $I^{\prime}$ whose union $I \cup I^{\prime}$ is an interval, $\mathrm{P}$ holds of $I \cup I^{\prime}$ iff it holds of each of $I$ and $I^{\prime}$

$$
\mathrm{P}\left(I \cup I^{\prime}\right) \Longleftrightarrow \mathrm{P}(I) \text { and } \mathrm{P}\left(I^{\prime}\right) \quad \text { whenever } I \cup I^{\prime} \text { is an interval. }
$$

The direction $\Longrightarrow$ makes $\mathrm{P}$ divisive (as illustrated by (2)), while its converse, $\Longleftarrow$, makes $\mathrm{P}$ cumulative (as illustrated by (3)). Clearly, any homgeneous predicate $\mathrm{P}$ 
is segmented. Conversely, a segmented predicate $P$ is homogeneous provided the linear order $\prec$ underlying the intervals is a subset of $U \times U$ for some finite set $U$. In the case of an $A$-string $\alpha_{1} \cdots \alpha_{n}$, repackaged as the $A$-model $\left\langle[n], S_{n},\left[\left[P_{a}\right]_{a \in A}\right\rangle\right.$, we can let

(a) $U$ be the set $[n]$ of string positions $1, \ldots, n$

(b) $\prec$ be the transitive closure of $S_{n}$

(c) each $a \in A$ name the homogeneous predicate $\mathrm{P}_{a}$ on intervals of $[n]$ such that

$$
\mathrm{P}_{a}(I) \Longleftrightarrow(\forall t \in I) t \in\left[\left[P_{a}\right]\right]
$$

for all intervals $I$ of $[n]$.

When convenient, we conflate a name $a \in A$ with the subset $\left[\left[P_{a}\right]\right]$ of $[n]$, and refer to $A$ as a set of subsets p of $U .^{2}$

Given an interval $I$ (such as $U$ ) and a set $A$ of subsets p of $I$, is there an $A$-string that depicts the predicates in $A$ over $I$, surveyed in increasing (chronological) order? To make this question precise, let us say

(a) a segmentation of $I$ is a string $I_{1} \cdots I_{n}$ of sub-intervals of $I$ such that

$$
I=\bigcup_{i=1}^{n} I_{i} \text { and }(\forall i \in[n-1])\left(\forall t \in I_{i}\right)\left(\forall t^{\prime} \in I_{i+1}\right) t \prec t^{\prime}
$$

(in words: a segmentation of $I$ is a finite ordered partition of $I$ )

(b) an $A$-depiction of $I$ is an $A$-string $\alpha_{1} \cdots \alpha_{n}$ such that for some segmentation $I_{1} \cdots I_{n}$ of $I$ and every $\mathrm{p} \in A, i \in[n]$, and $t \in \alpha_{i}$,

$$
\mathrm{p} \in \alpha_{i} \Longleftrightarrow t \in \mathrm{p}
$$

(making $\alpha_{1} \cdots \alpha_{n}$ a filmstrip of $A$ over $I$ ).

To illustrate, if $I$ is the closed unit interval $[0,1]=\{t \in \mathbb{R} \mid 0 \leq t \leq 1\}$ (over the real line $\mathbb{R}$ ) and $\mathrm{p}$ is the open unit interval $(0,1)=\{t \in \mathbb{R} \mid 0<t<1\}$, then $\square \mathrm{p} \mid$ is a $\{\mathrm{p}\}$-depiction of $I$, as is any string from the infinite set

$$
\square^{+} \square=\left\{\begin{array}{l|l|l|l|l|l|l|l}
\hline \mathrm{p} & \mathrm{p} \\
\hline & \mathrm{p} & \mathrm{p} & \square & \mathrm{p} & \mathrm{p} & \mathrm{p} & \ldots
\end{array}\right.
$$

2 Caution: the letter $\mathrm{p}$ is written in different fonts to distinguish its various uses as a picture $p$, a predicate/subset $\mathrm{P}$ or $\mathrm{p}$, and a predicate symbol $P_{a}$. While a predicate symbol can often be conflated with a predicate for simplicity (rebelling against logical pedantry), confusing a picture with either is somewhat more perilous. 
Pictorial narratives and temporal refinement

To formulate a useful necessary condition for the existence of an $A$-depiction of $I$, let us define for any $\mathrm{p} \in A$, a p-alternation in $I$ to be a string $t_{1} \cdots t_{n}$ of elements of $I$ such that for all $i \in[n-1], t_{i} \prec t_{i+1}$ and

$$
t_{i} \in \mathrm{p} \Longleftrightarrow t_{i+1} \notin \mathrm{p}
$$

(in words: a p-alternation is an increasing sequence of points in $I$ that alternate between being in and out of $\mathrm{p}$ ). Now, given any $A$-depiction $\alpha_{1} \cdots \alpha_{n}$ of $I$, its length $n$ is an upper bound on the length of any p-alternation in $I$. Let us say a subset $p$ of $I$ is $I$-stable if there is an integer $k$ such that for every p-alternation $t_{1} \cdots t_{n}$ in $I, n<k$ (bounding the length of any $\mathrm{p}$-alternation in $I$ by $k$ ). $I$-stability is not just necessary for an $A$-depiction of $I$ but sufficient.

Depiction theorem (Fernando 2016) Given an interval I and a finite set $A$ of subsets $\mathrm{p}$ of $I$, there is an A-depiction of I iff every $\mathrm{p}$ in $A$ is I-stable.

A special case of the depiction theorem is where every p-alternation $t_{1} \cdots t_{n}$ in $I$ has length $n \leq 1$ (for every $\mathrm{p} \in A$ ) so that the subset

$$
\{\mathrm{p} \in A \mid I \cap \mathrm{p} \neq \emptyset\}
$$

of $A$ (which we have been drawing as a box, and can be understood as a string of length 1) is an $A$-depiction of $I$. A somewhat more interesting case is where $I$ is a finite set $\left\{t_{1}, \ldots, t_{n}\right\}$ with $t_{1} \prec \cdots \prec t_{n}$, which we can turn into an $A$-depiction $\alpha_{1} \cdots \alpha_{n}$ of $I$ with

$$
\alpha_{i}:=\left\{\mathrm{p} \in A \mid t_{i} \in \mathrm{p}\right\} \quad \text { for each } i \in[n]
$$

albeit longer than necessary if say, $\alpha_{1}=\alpha_{2}$. (Note that the depiction theorem does not assume $I$ is finite.)

To pick out $A$-depictions uniquely, we compress strings as folows. A string $\alpha_{1} \cdots \alpha_{n}$ is stutterless if for all $i \in[n-1], \alpha_{i} \neq \alpha_{i+1}$. For any string $s=\alpha_{1} \cdots \alpha_{n}$, its block compression $b c(s)$ deletes every $\alpha_{i}$ such that $\alpha_{i}=\alpha_{i+1}$

$$
b c\left(\alpha_{1} \cdots \alpha_{n}\right):= \begin{cases}\alpha_{1} \cdots \alpha_{n} & \text { if } n<2 \\ b c\left(\alpha_{2} \cdots \alpha_{n}\right) & \text { if } \alpha_{1}=\alpha_{2} \\ \alpha_{1} b c\left(\alpha_{2} \cdots \alpha_{n}\right) & \text { otherwise. }\end{cases}
$$

Clearly, $b c(s)$ is stutterless, and

$$
s \text { is stutterless } \Longleftrightarrow b c(s)=s .
$$

Furthermore, for any $A$-depiction $s$ of $I, b c(s)$ is an $A$-depiction of $I$ that is shorter than any other $A$-depiction of $I$. Block compression $b c$ is a very direct implementation of 


\begin{tabular}{c|c|c|c|c} 
Dowty & Fillmore & Levin \& Rappaport Hovav & \multicolumn{2}{|l}{} \\
\hline BECOME & break & result (stative $a$ ) & $\bar{a}$ & $a$ \\
\hline DO & hit & manner (force $f$ ) & $\operatorname{ap}(f)$ & $\operatorname{ef}(f)$ \\
\hline
\end{tabular}

Table 1 Changes from Becoming to Doing

$(\mathrm{Ntc})_{A} \quad$ no time without change $A$

with change evaluated up to granularity $A$.

\subsection{From depicting to classifying change}

A rudimentary aspectual distinction widely recognized to be significant in temporality is that between statives and eventives (e.g., Kamp \& Reyle 1993). If a set $A$ consist of labels of stative predicates, an eventive is a predicate expressing change that can be represented up to granularity $A$ by an $A$-string. The simplest instances of change are formulated in Dowty (1979) through connectives DO and BECOME, that are associated in Table 1 with Levin \& Rappaport Hovav (2013)'s manner/result complementarity, which, in turn, generalizes the contrast between the verbs break and hit pointed out in Fillmore (1970). The fourth column of Table 1 effectively analyses BECOME as a function that maps a label $a$ to a string \begin{tabular}{|l|l|}
\hline $\bar{a}$ & $a$ \\
\hline
\end{tabular}

$$
\operatorname{BECOME}_{s}:=\lambda a \cdot \bar{a} \mid a
$$

where $\bar{a}$ labels a predicate $P_{\bar{a}}$ that negates the predicate $P_{a}$ labeled by $a$

$$
\forall x\left(P_{\bar{a}}(x) \equiv \neg P_{a}(x)\right) .
$$

The predicate logic formula (4), like the earlier formula

$$
\forall x\left(P_{p}(x) \equiv \bigvee_{v \in V} P_{(p, v)}(x)\right)
$$

from $\S 1.2$, belongs to the system $\mathrm{MSO}_{A}$ of Monadic Second-Order Logic over strings with a set $A$ of labels of unary predicates (e.g., Libkin 2004). As previously mentioned, the set $A$ varies over the set $\operatorname{Fin}(\Theta)$ of finite subsets of some large (infinite) set $\Theta$, assumed for present purposes to include labels $\operatorname{ap}(f)$ and $\operatorname{ef}(f)$ for the application and effects of certain forces $f$. The idea is that manners and results in Levin \& Rappaport Hovav (2013) are represented as forces and statives, respectively, with forces $f$ fed to the operator

$$
\mathrm{DO}_{s}:=\lambda f \cdot \operatorname{ap}(f) \mid \operatorname{ef}(f)
$$


Pictorial narratives and temporal refinement

\begin{tabular}{|c|c|c|}
\hline & atomic & extended \\
\hline+ conseq & $\begin{array}{l}\text { ACHIEVEMENT } \\
\text { culmination }\end{array}$ & $\begin{array}{l}\text { ACCOMPLISHMENT } \\
\text { culminated process }\end{array}$ \\
\hline STATIVE $a$ & \begin{tabular}{|l|l|}
$\bar{a}$ & $a$ \\
\end{tabular} & \begin{tabular}{|l|l|l|}
$\bar{a}, \operatorname{ap}(f)$ & $\bar{a}, \operatorname{ap}(f), \operatorname{ef}(f)$ & $\operatorname{ef}(f), a$
\end{tabular} \\
\hline - conseq & $\begin{array}{l}\text { (semelfactive) } \\
\text { point }\end{array}$ & $\begin{array}{l}\text { ACTIVITY } \\
\text { process }\end{array}$ \\
\hline$f$ & \begin{tabular}{l|l}
$\operatorname{ap}(f)$ & $\operatorname{ef}(f)$
\end{tabular} & \begin{tabular}{l|l|l|}
$\operatorname{ap}(f)$ & $\operatorname{ap}(f), \operatorname{ef}(f)$ & $\operatorname{ef}(f)$
\end{tabular} \\
\hline
\end{tabular}

Table 2 Moens \& Steedman (1988)'s reconstruction of ARKV with strings

just as statives $a$ are fed to $\mathrm{BECOME}_{s}$. The obvious question is:

what do ap $(f), \operatorname{ef}(f)$ mean and what are we to make of the sortal difference between statives $a$ and forces $f$ ?

Answering this question will take up most of the remainder of this section (and some of the next).

Part of the answer can be gleaned from the Aristotle-Ryle-Kenny-Vendler verb classification (Dowty 1979), reworked in Table 2 according to Moens \& Steedman (1988). Notice that the fourth column of Table 1 appears under the feature atomic in Table 2, to the left of the (contrary) feature extended. Whereas $\bar{a}$ is precluded from occuring in a box where $a$ occurs (by (4), the labels ap $(f)$ and ef $(f)$ occur together in the second box under the extended column. To understand what ap $(f)$ and $\operatorname{ef}(f)$ might mean, it is instructive to ask: where is the force that, under the principle (Inr) of inertia, effects the change from $\bar{a}$ to $a$ depicted by $\bar{a}|a|$ ? That force certainly does not appear in $\operatorname{BECOME}_{s}(a)$ - at least not explicitly.

We can pick out string positions $x$ where $a$ does not occur but is succeeded by a position where $a$ occurs, using the $\mathrm{MSO}_{\{a\}}$-formula $\chi_{l(a)}(x)$

$$
\begin{aligned}
\chi_{l(a)}(x):= & \exists y\left(x S y \wedge P_{a}(y)\right) \wedge \neg P_{a}(x) \\
& a \text { occurs at the position next to } x \text { but not at } x
\end{aligned}
$$

(making $l(a)$ a left border of $a$ ). To illustrate, if the positions of a string $s=\alpha_{1} \cdots \alpha_{n}$ picked out by $\chi_{l(a)}(x)$ are put in

$$
\begin{aligned}
\mathrm{p}_{l(a)} & :=\left\{i \in[n]|s|=\chi_{l(a)}[x / i]\right\} \\
& =\left\{i \in[n-1] \mid a \in \alpha_{i+1}-\alpha_{i}\right\}
\end{aligned}
$$

then for $s=$\begin{tabular}{|l|l|l|l|l|}
$a^{\prime}$ & $a$ & $a, a^{\prime}$ & $a^{\prime}$ & $a$ \\
\hline
\end{tabular}$($ with $n=5)$,

$$
\mathrm{p}_{l(a)}=\{1,4\} \text { and } \mathrm{p}_{l\left(a^{\prime}\right)}=\{2\} .
$$


For each $a \in A$, we can treat $l(a)$ as a label in $\Theta$ that we can introduce into an $A$-string $\alpha_{1} \cdots \alpha_{n}$ by expanding it to the $(A \cup\{l(a) \mid a \in A\})$-string $\beta_{1} \cdots \beta_{n-1} \alpha_{n}$ where

$$
\beta_{i}:=\alpha_{i} \cup\left\{l(a) \mid a \in \alpha_{i+1}-\alpha_{i}\right\} \text { for } i \in[n-1] .
$$

For example, \begin{tabular}{|l|l|l|l|l|l|l|l|l|l|l|}
$a^{\prime}$ & $a$ & $a, a^{\prime}$ & $a^{\prime}$ & $a$ & expands to $a^{\prime}, l(a)$ & $a, l\left(a^{\prime}\right)$ & $a, a^{\prime}$ & $l(a), a^{\prime}$ & $a$ & \\
\hline
\end{tabular} emphasize what BECOME and DO have in common, let us modify BECOME $\mathrm{BD}_{s}$ to

$$
\begin{aligned}
\operatorname{BECOME}_{s}^{\prime} & :=\lambda a \cdot l(a) a \\
\left(\text { compare to } \text { DO }_{s}\right. & :=\lambda f \cdot \operatorname{ap}(f) \operatorname{ef}(f)
\end{aligned}
$$

making $l(a)$ the BECOME-analog of ap $(f)$, and $a$ the BECOME-analog of ef $(f)$. Or zeroing in on the labels, we can reformulate $\mathrm{BECOME}_{s}$ and $\mathrm{DO}_{s}$ as operators

$$
\operatorname{BECOME}:=\lambda a . l(a) \quad \text { DO }:=\lambda f . \mathrm{ap}(f)
$$

that return labels (in $\Theta$ ). The label $l(a)$ expresses change (formulated in $\chi_{l(a)}(x)$ ) which neither $a$ nor $\bar{a}$ does. (Note that the constraint (4) introducing $\bar{a}$ has no use for the successor relation symbol $S$.) What change ap $(f)$ expresses, we leave for the next subsection.

In the meantime, let us pause to systematise the expansions of $A$ considered above, paying particular attention to their order independence. For $b \notin A$ and an $\mathrm{MSO}_{A}$-formula $\chi_{b}(a)$, let $c_{A}^{b}:\left(2^{A}\right)^{*} \rightarrow\left(2^{A \cup\{b\}}\right)^{*}$ be the function mapping an $A$ string $\alpha_{1} \cdots \alpha_{n}$ to the $(A \cup\{b\})$-string

$$
\beta_{1} \cdots \beta_{n} \text { where } \beta_{i}:=\alpha_{i} \cup\left\{b \mid \alpha_{1} \cdots \alpha_{n} \models \chi_{b}[x / i]\right\} \text { for } i \in[n] .
$$

For example, if $b=\bar{a}$ and $\chi_{\bar{a}}(x)$ is $\neg P_{a}(x)$ then

$$
c_{\{a\}}^{\bar{a}}(\square \mid a)=\bar{a} \mid a
$$

and if $b=l(a)$ then for the previously defined $\operatorname{MSO}_{\{a\}}$-formula $\chi_{l(a)}(x)$,

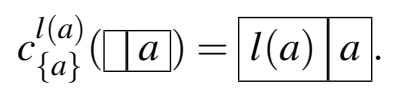

Given another label $b^{\prime} \notin A \cup\{b\}$ and an $\mathrm{MSO}_{A^{-}}$-formula $\chi_{b^{\prime}}(x)$, we may expect

$$
c_{A \cup\{b\}}^{b^{\prime}}\left(c_{A}^{b}(s)\right)=c_{A \cup\left\{b^{\prime}\right\}}^{b}\left(c_{A}^{b^{\prime}}(s)\right)
$$

for any $A$-string $s$. In fact, rather than adding a label one at a time to $A$, we might do so in one go. Given a set $B$ disjoint from $A$ and an $\mathrm{MSO}_{A}$-formula $\chi_{b}(x)$ for each $b \in B$, let $e_{A}^{B}:\left(2^{A}\right)^{*} \rightarrow\left(2^{A \cup B}\right)^{*}$ map an $A$-string $\alpha_{1} \cdots \alpha_{n}$ to the $(A \cup B)$-string

$$
\beta_{1} \cdots \beta_{n} \text { where } \beta_{i}:=\alpha_{i} \cup\left\{b \in B \mid \alpha_{1} \cdots \alpha_{n} \models \chi_{b}[x / i]\right\} \text { for } i \in[n] .
$$


Pictorial narratives and temporal refinement

To see that

$$
e_{A}^{\left\{b, b^{\prime}\right\}}(s)=c_{A \cup\{b\}}^{b^{\prime}}\left(c_{A}^{b}(s)\right)=c_{A \cup\left\{b^{\prime}\right\}}^{b}\left(c_{A}^{b^{\prime}}(s)\right),
$$

let us invert the function $e_{A}^{B}$, defining the $A$-reduct $\rho_{A}(s)$ of any string $s$ of sets (which need not be a subset of $A$ ) to be the componentwise intersection of $s$ with $A$

$$
\rho_{A}\left(\alpha_{1} \cdots \alpha_{n}\right):=\left(\alpha_{1} \cap A\right) \cdots\left(\alpha_{n} \cap A\right) \text {. }
$$

For example,

$$
\rho_{\{a\}}\left(\begin{array}{l|l|}
\hline l(a) & a
\end{array}\right)=\square a
$$

and for any $A$-string $s, \rho_{A}(s)=s$ and

$$
\rho_{A}\left(e_{A}^{B}(s)\right)=s \text { for } B \cap A=\emptyset .
$$

Fact Let $A$ and $B$ be disjoint sets, and each $b \in B$ label an $M_{S S} O_{A}$-formula $\chi_{b}(x)$. Then for any $(A \cup B)$-string $s, s=e_{A}^{B}\left(\rho_{A}(s)\right)$ iff for every $b \in B$, s satisfies

$$
\forall x\left(P_{b}(x) \equiv \chi_{b}(x)\right)
$$

Explicit definitions (5) include (1) and (4) and the instance given by $b=l(a)$ above. The fact follows from a fundamental property of reducts

$(\dagger)$ for all sets $A$ and $A^{\prime}$ such that $A \subseteq A^{\prime}$, for every $\mathrm{MSO}_{A^{-}}$-sentence $\varphi$ and every $A^{\prime}$-string $s$,

$$
s \models \varphi \Longleftrightarrow \rho_{A}(s) \models \varphi .
$$

$(\dagger)$ is an instance of the satisfaction condition in Goguen \& Burstall (1992) characteristic of an institution, a logical system varying $A$ smoothly. More below.

\subsection{Open-ended refinements and lexicalized meaning}

Returning to our initial example of picture $p$ labeling a predicate $P_{p}$ defined through a set of viewpoints $v$ that pair with $p$ for predicates $P_{(p, v)}$, let us sharpen (1) slightly to

$$
\forall x\left(P_{p}(x) \equiv \bigvee_{v \in V(p)} P_{(p, v)}(x)\right)
$$

tying the set $V(p)$ of viewpoints $v$ to $p$, as different pictures may involve different viewpoints, and $V(p)$ had better be finite for the disjunction to fall within $\mathrm{MSO}_{\{p\} \times V(p)}$. Notice that $v$ (as well as $p$ ) can be construed as a discourse referent 
subject to different interpretations. Hence, we are free to throw into $V(p)$ a wildcard $\star$ for an otherwise clause $P_{(\star, p)}(x)$ completing the disjunction (and securing the biconditional $\equiv)$. The idea is that $\star$ represents the limits of our knowledge, our ignorance, the unknown. Alternatively, we might break the biconditional (1) into the conditionals

$$
\forall x\left(P_{(p, v)}(x) \supset P_{p}(x)\right)
$$

for each $v \in V$ (without requiring $\star \in V$ ).

We move now from viewpoints behind pictures to forces $f$ behind change (on which time depends according to $(\mathrm{Ntc}))$. Let us write $\mathrm{ap}_{f}$ for the unary predicate symbol $P_{\hat{a}}$ where $\hat{a}$ is $\operatorname{ap}(f)$ (saying $f$ is applied), and ef $f$ for $P_{\hat{a}}$ where $\hat{a}$ is $\operatorname{ef}(f)$ (saying the effects of $f$ hold). Next, given a label $a$ for a stative predicate symbol $P_{a}$, can we link the left border $l(a)$ to forces, as in

$$
\forall x\left(P_{l(a)}(x) \equiv \bigvee_{f \in F(a)} \operatorname{ap}_{f}(x)\right)
$$

for a suitable finite set $F(a)$ of forces $f$ for $a$ (with perhaps $a$ as $\operatorname{ef}(f)$, and, if necessary, a wildcard $\star_{a}$ in $\left.F(a)\right)$ ? Not so fast. There is more to $l(a)$ than applying a force; $a$ is also understood to hold following some such application, as spelled out in

$$
\chi_{l(a)}(x):=\exists y\left(x S y \wedge P_{a}(y)\right) \wedge \neg P_{a}(x)
$$

from the previous subsection. The problem is that other forces applied at the same time as $f$ can interfere with $\operatorname{ef}(f)$. In the absence of such interference, we may step from ap $(f)$ to ef $(f)$, as expressed in the implication

$$
\forall x \forall y\left(x S y \wedge \operatorname{ap}_{f}(x) \wedge \neg \operatorname{opposed}_{f}(x) \supset \operatorname{ef}_{f}(y)\right)
$$

where opposed $f(x)$ is a formula saying some force opposing $f$ is applied at $x$

$$
\operatorname{opposed}_{f}(x):=\bigvee_{f^{\prime} \in O(f)} \operatorname{ap}_{f^{\prime}}(x)
$$

for some finite set $O(f)$ of $f$-opposing forces $f^{\prime}$ that clash with $f$ in that

$$
\neg \exists x\left(\operatorname{ef}_{f}(x) \wedge \operatorname{ef}_{f^{\prime}}(x)\right)
$$

(and, if necessary, a wildcard $\star_{f} \in O(f)$ to keep the set finite). This is not to say that $\neg \operatorname{opposed}_{f}(x)$ is necessary to conclude $\mathrm{ef}_{f}(y)$ from $x S y \wedge \operatorname{ap}_{f}(x)$; only that $\operatorname{opposed}_{f}(x)$ requires further assumptions to draw that conclusion. While (6) is problematic, we can count on one of its directions (from left to right) for the instance

$$
\forall x \forall y\left(x S y \wedge P_{a}(y) \wedge \neg P_{a}(x) \supset \bigvee_{f \in F(a)} \operatorname{ap}_{f}(x)\right)
$$


Pictorial narratives and temporal refinement

of inertia, no change $l(a)$ without some force from $F(a)$.

Whereas the predicates $P_{\bar{a}}$ and $P_{l(a)}$ labeled in

$$
\operatorname{BECOME}_{s}=\lambda a \cdot \bar{a} \mid a \text { and } \operatorname{BECOME}=\lambda a \cdot l(a)
$$

are defined by $P_{a}$ through instances of (5) given by $b=\bar{a}, l(a)$, there is no predicate $P_{f}$ that defines the predicates ap and $_{f}$ labeled in

$$
\mathrm{DO}_{s}=\lambda f \cdot \operatorname{ap}(f) \operatorname{ef}(f) \text { and } \mathrm{DO}=\lambda f \cdot \operatorname{ap}(f) .
$$

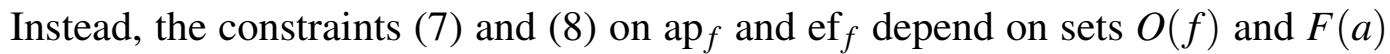
that vary with the circumstances of the utterance. Returning to Table 1, we might say, echoing Levin \& Rappaport Hovav (2013), that

(a) a result (break/BECOME) verb lexically prescribes its post-condition $a$ (as well as its pre-condition $\bar{a}$ ), while its manner is supplied by context of use

(b) a manner (hit/DO) verb lexically prescribes the manner $f$, leaving context to make what it will of $\operatorname{ef}_{f}, \operatorname{ap}_{f}{ }^{3}$

The division of labour in meaning between the lexicon and context of use carries over to a picture $p$; its "lexicalized meaning" $P_{p}$ decouples $p$ as an artifact from the viewpoint $v$ and the other circumstances of its creation/use (recall footnote 1 in the introduction). We can sharpen the predicate $P_{p}$ to some predicate $P_{(p, v)}$ or $P_{(p, \vec{z})}$ incorporating the circumstances $\vec{z}$ of projection (which need not be in some objective world, but rather in the mind of an agent $z$, as in the second picture of a free perception pair in Abusch \& Rooth (2017)). There is no limit to the labels we may put into the infinite set $\Theta$, a finite subset of which is chosen for $A$. This paper's focus has not been on any projection (which reduces the alphabet $2^{A}$ of an $A$-string), but rather on temporal constraints between different boxes in an $A$-string, expressed in $\chi_{l(a)}$ and (7), (8) through the successor relation $S$ holding a string together.

\section{String iconicity: projections, parts and limitations}

For string iconicity, it is helpful to construe a string two ways, as

(a) a semantic entity/model, and

(b) a syntactic expression/representation.

3 That post-condition, pre-condition pair may well describe an incremental change over some scale, expanding $A$ so that ap $(f)$ and $\operatorname{ef}(f)$ may occur together in a box, as in the extended column of Table 2 above (Fernando 2019). 
Fernando

\begin{tabular}{|c|c|c|c|c|}
\hline predicate & & & picture & automata \\
\hline stative & holds (be) & static & still (snapshot) & state \\
eventive & happens (do/become) & dynamic & movie (motion pic) & transition \\
\hline
\end{tabular}

Table 3 Statives $v s$ eventives pictured

For example, a regular expression $e$ over an alphabet $\Sigma$ denotes a set $\mathscr{L}(e) \subseteq \Sigma^{*}$ of strings over $\Sigma$, with

$$
\mathscr{L}(s)=\{s\} \text { for any } s \in \Sigma^{*} .
$$

If a string $s=\sigma_{1} \cdots \sigma_{n} \in \Sigma^{*}$ is identified with the $\mathrm{MSO}_{\Sigma}$-model $\left\langle[n], S_{n},\left[P_{\sigma}\right]_{\sigma \in \Sigma}\right\rangle$ that interprets $P_{\sigma}$ as $\left[P_{\sigma}\right]:=\left\{i \in[n] \mid \sigma=\sigma_{i}\right\}$ then a theorem due to Büchi, Elgot and Trakhtenbrot (BET) states that there is an $\mathrm{MSO}_{\Sigma}$-sentence $\varphi$ such that

$$
\mathscr{L}(e)=\left\{s \in \Sigma^{*} \mid s \models \varphi\right\}
$$

and conversely, for any $\mathrm{MSO}_{\Sigma}$-sentence $\varphi,(10)$ holds for some regular expression $e$ (e.g., Libkin 2004: 124). (That is, BET says $\mathrm{MSO}_{\Sigma}$-sentences pick out exactly the regular languages over $\Sigma$.) The degree of resemblance, if any, between an MSOsentence $\varphi$ and a string that satisfies $\varphi$ contrasts starkly with (9). The aim of this section is to extend (9) to further iconic representations, where the form-meaning resemblance is spelled out by projections and mereological relations on strings. To define these functions and parts, we work with $A$-strings $\alpha_{1} \cdots \alpha_{n}$ (over the alphabet $\Sigma=2^{A}$ ), understood as $\mathrm{MSO}_{A^{A}}$-models $\left\langle[n], S_{n},\left[\left[P_{a}\right]\right]_{a \in A}\right\rangle$, rather than $\mathrm{MSO}_{2^{A}}$-models $\left\langle[n], S_{n},\left[P_{\sigma}\right]_{\sigma \subseteq A}\right\rangle$. As in the previous section, an element $a$ of $A$ may label a stative predicate $P_{a}$ that holds of a stretch of time, or, in the case of $l(a)$, a non-stative predicate $P_{l(a)}$ that happens just before the succeeding stretch, described as eventive in Table 3. The stative/eventive distinction is reflected in the projections defined below.

\subsection{Projections from reducts and compressing two ways}

Just as any set $\Theta$ is the union of the set $\operatorname{Fin}(\Theta)$ of its finite subsets, any linear order $<$ on $\Theta$ can be constructed from its finite chains $a_{1}<\cdots<a_{n}$, which for $A=\left\{a_{1}, \ldots, a_{n}\right\}$, we can formulate as the $A$-string $a_{1} \cdots a_{n}$ or $<_{A}$ for short. To make this construction precise, we define for every finite subset $A$ of $\Theta$, the function $d_{A}:\left(2^{\Theta}\right)^{*} \rightarrow\left(2^{A}\right)^{*}$ mapping a $\Theta$-string $s$ to the $A$-string $d_{A}(s)$ obtained from the $A$-reduct of $s$ by deleting all occurrences of the empty box $\square$; i.e., $d_{A}(s)$ is $\mathrm{d}_{\square}\left(\rho_{A}(s)\right)$, 
Pictorial narratives and temporal refinement

\begin{tabular}{c|c} 
eventives & statives \\
\hline $\mathrm{d}_{\square}(s):=s$ without $\square$ & $b c(s):=s$ without stutters \\
$s \square s^{\prime} \rightsquigarrow s s^{\prime}$ (non-abutting) & $s \alpha \alpha s^{\prime} \rightsquigarrow s \alpha s^{\prime}$ (abutting)
\end{tabular}

Table 4 Compressing two ways (with abutment from cumulativity)

where $\mathrm{d}_{\square}(\varepsilon):=\varepsilon$ and

$$
\mathrm{d}_{\square}(\alpha s):= \begin{cases}\mathrm{d}_{\square}(s) & \text { if } \alpha=\square \\ \alpha \mathrm{d}_{\square}(s) & \text { otherwise. }\end{cases}
$$

To illustrate, for $\Theta=\mathbb{R}$ and $A=\{2, \pi, 1,7\},<_{A}$ is \begin{tabular}{|l|l|l|l|}
\hline 1 & 2 & $\pi$ & 7
\end{tabular} and

$$
d_{A}\left(<_{A \cup\{5,-1\}}\right)=\mathrm{d}_{\square}\left(\rho_{A}\left(\begin{array}{l|l|l|l|l|l}
\hline-1 & 1 & 2 & \pi & 5 & 7
\end{array}\right)\right)=\mathrm{d}_{\square}\left(\begin{array}{l|l|l|l|l|}
\hline & 1 & 2 & \pi & 7
\end{array}\right)=<_{A} .
$$

Now, we can describe $<$ as the inverse limit of the $A$-strings $<_{A}$ and functions $d_{A}$, noting that $\left\{d_{A}\right\}_{A \in F i n(\Theta)}$ is a projective system in that for any $A \in \operatorname{Fin}(\Theta)$,

(a) $\quad d_{A}(s)=s$ for any $A$-string $s$, and

(b) $\quad d_{B}(s)=d_{B}\left(d_{A}(s)\right)$ for any $B \subseteq A$ and any $\Theta$-string $s$.

Next, we consider how these projections $d_{A}$ relate to the depictions and expansions from the previous section, where in place of $\mathrm{d}_{\square}$, we have block compression $b c$ for the abutment required by interval segementations $(\$ 2.1)$; see Table 4 . These two ways to compress can be related by pairing the left border $l(a)$ with the right border $r(a)$

$$
\chi_{r(a)}(x):=P_{a}(x) \wedge \neg \exists y\left(x S y \wedge P_{a}(y)\right)
$$

$a$ occurs at $x$ but not at any following position

and defining the border translation $b_{A}(s)$ of an $A$-string $s$ to be $\rho_{B}\left(e_{A}^{B}(s)\right)$ where $B$ is the set

$$
A_{\bullet}:=\{l(a) \mid a \in A\} \cup\{r(a) \mid a \in A\}
$$

of borders of $A$ (with $\chi_{l(a)}$ making $a$ true, and $\chi_{r(a)}$ stopping $a$ from being true). For instance,

$$
\begin{aligned}
& b_{\left\{a, a^{\prime}\right\}}\left(\begin{array}{l|l|l|l|l|}
\hline & a & a, a^{\prime}
\end{array}\right)=\rho_{B}\left(\begin{array}{ll|l}
l(a) & a, l\left(a^{\prime}\right) & a, a^{\prime}, r(a), r\left(a^{\prime}\right)
\end{array}\right) \text { where } B \text { is }\left\{a, a^{\prime}\right\} \bullet \\
& =l(a) l\left(a^{\prime}\right) \mid r(a), r\left(a^{\prime}\right) \text {. }
\end{aligned}
$$


Looking through $b_{A}$, we see that $b c$ and $\mathrm{d}_{\square}$ are opposite sides of the same coin

$$
b_{A}(b c(s))=\mathrm{d}_{\square}\left(b_{A}(s)\right) \text { for any } A \text {-string } s \text { that does not end in } \square \text {. }
$$

Projection with respect to $A$ takes the $A$-reduct before compressing

$$
f_{A}(s):=f\left(\rho_{A}(s)\right) \text { for any string } s \text { of sets }
$$

where $f=b c$ if $A$ labels statives, and $f=\mathrm{d}_{\square}$ if $A$ labels eventives. $A$-projections $f_{A}$ provide interesting examples of Goguen \& Burstall (1992)'s institutions, courtesy of the biconditional

$$
s \in f_{A}^{-1} L \Longleftrightarrow f_{A}(s) \in L
$$

for any $\Theta$-string $s$ and set $L$ of $A$-strings. If the string $s$ in (11) is understood as a model, and the language $L$ is the denotation set $\left[[\varphi] \subseteq\left(2^{A}\right)^{*}\right.$ of an expression $\varphi$, then (11) becomes the satisfaction condition

$$
s=\left\langle f_{A}\right\rangle \varphi \Longleftrightarrow f_{A}(s) \models \varphi
$$

where $\left\langle f_{A}\right\rangle$ is the modal operator with accessibility relation $f_{a}$. In case $[[\varphi]]$ is a singleton set $\left\{s^{\prime}\right\}$, the right side of (12) reduces to the equation $f_{A}(s)=s^{\prime}$.

\subsection{Parts and partiality}

As $f_{A}$ is a function, the modal operator $\left\langle f_{A}\right\rangle$ in (12) can be read existentially as a diamond or universally as a square. The difference takes on significance when $f_{A}$ is weakened to a mereological relation such as subsumption $\unrhd$, a form of conjunction elimination for strings

$$
\alpha_{1} \cdots \alpha_{n} \unrhd \alpha_{1}^{\prime} \cdots \alpha_{m}^{\prime} \Longleftrightarrow n=m \text { and } \alpha_{i}^{\prime} \subseteq \alpha_{1} \text { for all } i \in[n]
$$

non-deterministically generalizing reducts

$$
s \unrhd \rho_{A}(s) \text { for all } A \text { and strings } s \text { of sets. }
$$

$\unrhd$ is useful for variable-free (iconic?) formulations of constraints such as inertia (8)

$$
\begin{array}{l|l|l|}
\hline \bar{a} & a & \operatorname{ap}\left(F_{a}\right) \\
\hline
\end{array}
$$

via an implication operator $\Rightarrow$ on string sets (e.g., Fernando 2008: 329-332).

Beyond $A$-strings and the semantic timelines they represent, there are more elaborate structures that move into pragmatic territory, notably (a) record types encoding interaction centered around questions under discussion (e.g., Cooper \& Ginzburg 2015), and (b) Segmented Discourse Representation Structures supporting deviations from temporal succession based on maximizing discourse coherence (e.g., Asher \& Lascarides 2003). Implicit in the attention paid to $A$-strings and finite-state methods above is the challenge of doing a bit with less (and understanding what that is and can be). This is not to deny the need for more to do more. 
Pictorial narratives and temporal refinement

\section{References}

Abusch, D. 2013. Applying discourse semantics and pragmatics to co-reference in picture sequences. In Proceedings of Sinn und Bedeutung 17, 9-25. Paris.

Abusch, D. 2014. Temporal succession and aspectual type in visual narrative. In L. Crnič \& U. Sauerland (eds.), The Art and Craft of Semantics: A Festschrift for Irene Heim, vol. 1, 9-29. MIT Working Papers in Linguistics 70.

Abusch, D. \& M. Rooth. 2017. The formal semantics of free perception in pictorial narratives. In Proceedings of the 21st Amsterdam Colloquium, 85-95. University of Amsterdam.

Asher, N. \& A. Lascarides. 2003. Logics of Conversation. Cambridge University Press.

Cooper, R. \& J. Ginzburg. 2015. TTR for natural language semantics. In S. Lappin \& C. Fox (eds.), Handbook of Contemporary Semantic Theory, 375-407. WileyBlackwell 2nd edn.

Davidson, D. 1967. The logical form of action sentences. In N. Rescher (ed.), The Logic of Decision and Action, 81-95. University of Pittsburgh Press.

Dowty, D.R. 1979. Word Meaning and Montague Grammar. Reidel, Dordrecht. DOI 10.1007/978-94-009-9473-7.

Dowty, D.R. 1986. The effects of aspectual class on the temporal structure of discourse: semantics or pragmatics? Linguistics and Philosophy 9(1). 37-61.

Fernando, T. 2008. Branching from inertia worlds. Journal of Semantics 25. 321-44.

Fernando, T. 2016. Prior and temporal sequences for natural language. Synthese 193(11). 3625-3637.

Fernando, T. 2019. Projecting temporal properties, events and actions. In Proceedings 13th International Conference on Computational Semantics, 1-12. www . aclweb .org/anthology/W19-0401.

Fillmore, C.J. 1970. The grammar of hitting and breaking. In R.A. Jacobs \& P.S. Rosenbaum (eds.), Readings in English Transformational Grammar, 120-133. Ginn.

Goguen, J.A. \& R.M. Burstall. 1992. Institutions: abstract model theory for specification and programming. Journal of the ACM 39(1). 95-146.

Greenberg, G. 2013. Beyond resemblance. Philosophical Review 122(2). 215-287.

Hagen, M.A. 1986. Varieties of Realism: Geometries of Representational Art. Cambridge university Press.

Kamp, H. 2013. The time of my life. https://lucian.uchicago.edu/blogs/ elucidations/files/2013/08/Kamp_TheTimeOfMyLife.pdf.

Kamp, H. \& U. Reyle. 1993. From Discourse to Logic. Kluwer. DOI 10.1007/97894-017-1616-1.

Levin, B. \& M. Rappaport Hovav. 2013. Lexicalized meaning and manner/result 
complementarity. In B. Arsenijević, B. Gehrke \& R. Marín (eds.), Subatomic Semantics of Event Predicates, 49-70. Springer.

Libkin, L. 2004. Elements of Finite Model Theory. Springer. DOI 10.1007/978-3662-07003-1.

Maier, E. \& S. Bimpikou. To appear. Shifting perspectives in pictorial narratives. In Proceedings Sinn und Bedeutung 23, Barcelona.

Moens, M. \& M. Steedman. 1988. Temporal ontology and temporal reference. Computational Linguistics 14(2). 15- 28.

Perniss, P. \& G. Vigliocco. 2014. The bridge of iconicity: from a world of experience to the experience of language. Phil. Trans. R. Soc. B 369. DOI 10.1098/rstb.2013.0300.

Rooth, M. \& D. Abusch. 2018. Picture descriptions and centered content. In Proceedings Sinn und Bedeutung 21, 1051-1064. University of Edinburgh.

Shin, S-J. 2015. The mystery of deduction and diagrammatic aspects of representation. Rev. Phil. Psych. 6. 49-67. DOI 10.1007/s13164-014-0212-5.

Computer Science Department

Trinity College Dublin, Ireland

Tim.Fernando@tcd.ie 